\title{
Spatial Load Prediction Considering Spatiotemporal Distribution of Electric Vehicle Charging Load
}

\author{
Xiang $\mathrm{Gao}^{1}$, Lingyan $\mathrm{We}^{2 *}$, Bing $\mathrm{Wang}^{2}$, Guiru $\mathrm{Chen}^{2}$, and $\mathrm{Xiaoyue}_{\mathrm{Wu}}^{2}$ \\ ${ }^{1}$ College of Electrical Engineering, Nanjing Institute of Industry Technology, Nanjing, Jiangsu Province, 210023, China \\ ${ }^{2}$ College of Energy and Electrical Engineering, Hohai University, Nanjing, Jiangsu Province, 211100, China
}

\begin{abstract}
In view of the influence of large-scale electric vehicle access to the distribution network on spatial load prediction, this paper proposes a spatial load prediction method for urban distribution network considering the spatial and temporal distribution of electric vehicle charging load. Firstly, electric vehicles are classified according to charging mode and travel characteristics of various types of vehicles. Secondly, the probability distribution function is fitted to the travel rules of electric vehicles according to the travel survey and statistical data of residents. Then, the model of electric vehicle travel chain is constructed, and the charging load in different regions and different times is calculated by Monte Carlo method. Finally, based on the actual data of a certain area, the predicted spatial load values of different functional communities in one day are obtained, which can provide reference for future urban distribution network planning.
\end{abstract}

\section{Introduction}

Spatial load prediction, also known as district load forecasting, was first proposed and perfected by H.L.Willis of the United States in the 1980s[1]. It can be used to predict the geographical distribution and numerical value of the load in the planning area, and is an important reference for power grid planning and construction[2]. The main methods used in spatial load prediction are trend method, multivariable method, load density index method and land use simulation method. Literature[3-5] adopted land use simulation method to realize spatial load prediction; reference[6-8] adopted load density index method to realize spatial load prediction, but these methods did not consider the impact of large-scale EVs access on spatial load forecasting results. EV is the main development direction of new energy vehicles in the future. In recent years, it has become a hot spot in the development of automobile industry at home and abroad. The national policy guidance is obvious, the development speed is accelerating, and it will gradually enter the industrialization stage[9-10]. Due to the uncertainty and difference of the demand and behaviour of EV users, the future large-scale EV charging load has the characteristics of randomness, intermittence and volatility in time and space[11], which will bring difficulties to the safe operation and optimal dispatching of the power grid[12]. Theoretical support for the research on the regulation strategy of EVs widely connected to the power grid is provided in this paper.
EV load forecasting is the basis of the development of EVs. A lot of research has been done on the charging load forecasting of EVs at home and abroad. Basically, Monte Carlo simulation algorithm is used to calculate the charging load. Among them, reference[13] divides EVs into private cars, buses and taxis for analysis and modeling, but the selection of initial state of charge and initial charging time distribution of vehicles has certain subjectivity. Reference[14] established the probability distribution model of the trip end time and the daily mileage by using the statistical method, and considered that the trip end time is equal to the starting charging time. However, it is not reasonable for EVs with a daily charging frequency greater than once. Most of the researches on the appeal documents ignore the randomness of EV charging load in time and space. The forecasting of EVs travel distribution and the diversity of charging places are not considered carefully, and the forecasting method is not mature enough.

In response to the above problems, combined with the future development of EVs in Chinese cities, a spatial load forecasting method considering the temporal and spatial distribution of EV charging load power is proposed. The influencing factors of EV charging load are analyzed, and the travel chain of EV users is considered. It can more accurately simulate the spacetime transfer characteristics of EVs. Finally, the feasibility and effectiveness of this method are verified by taking the electricity load of a certain urban area as an example. 


\section{The Influence Factor of EV Charging Load}

The load of EV will be affected by many factors, so its size and spatial distribution characteristics are determined by these factors, such as the performance of EVs, users' habits, charging environment, use conditions and so on. In order to predict the spatial and temporal distribution of EV charging load, the following factors need to be considered: the type of EVs, the starting time of EV journey, the mileage of EVs, the parking time, the charging mode.

\subsection{Types of EVs}

At present, EVs are mainly used in private cars, taxis and buses. The driving route and time are usually fixed for electric buses, so the charging locations are easy to master; for private cars and taxis, their driving routes are random, and the charging locations are not fixed. Therefore, for different types of EVs, the location and size of charging load are different.

\subsection{Start Time of EV Journey}

For a car, it is obvious that there is a close relationship between the start time of the daily trip and the time distribution of the charging demand. Based on the statistical results of the 2017 National Household Travel Survey (NHTS), this paper extracts the travel start time of private cars in one day to study the operation characteristics of EVs.

Using the actual data, the probability distribution of the start time of the electric private car journey can be obtained. The probability distribution of users' journey start time is shown in Figure 1.

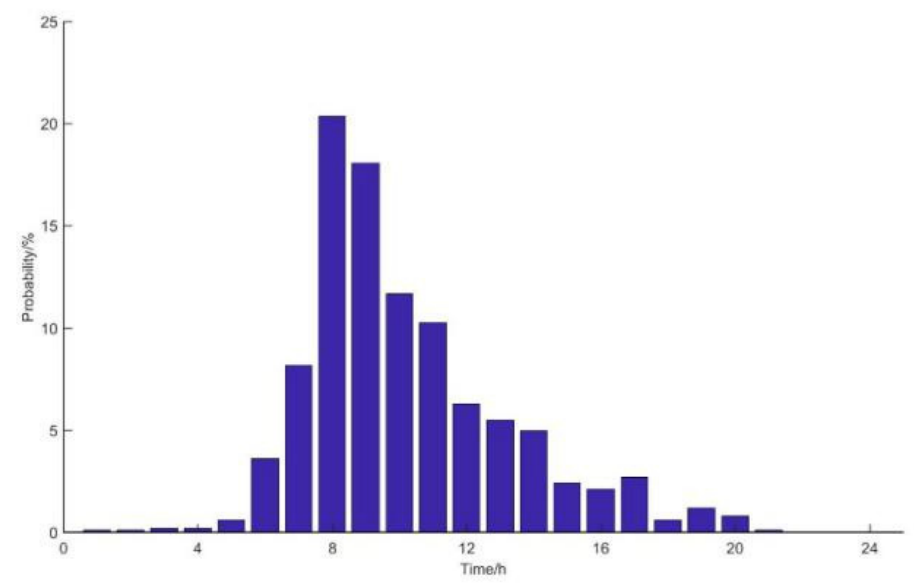

Figure. 1. Probability distribution of users' journey start time.

Fitting the probability distribution, it is found that the probability of the start time of the users' journey approximately obeys the normal distribution and the probability density function is

$$
f(x)=\frac{1}{x \sigma \sqrt{2 \pi}} \exp \left[-\frac{(x-\mu)^{2}}{2 \sigma^{2}}\right]
$$

Among them, $\mu$ is the expected value of the travel start time, and $\sigma$ is the standard deviation. After fitting, $\mu=7.89, \sigma=1.96$.

\subsection{The Driving Mileage of EVs}

The charging frequency and the time required for each charging of EV users are closely related to mileage. For different users, due to their different car habits, there are obvious differences in mileage. At the same time, the driving mileage of different types of EVs is also quite different. The private cars are mainly used for commuting and entertainment, so the driving mileage is short and regular.

According to the statistical results of NHTS in 2017, the distance of each segment of electric private cars approximately obeys lognormal distribution, and its probability density function is

$$
f(x)=\frac{1}{x \sigma \sqrt{2 \pi}} \exp \left[-\frac{(\ln x-\mu)^{2}}{2 \sigma^{2}}\right]
$$

Among them, $\mu$ is the expected value of travel distance, and $\sigma$ is the standard deviation. After fitting, $\mu=3.68, \sigma=0.88$.

For taxis, the daily mileage is the longest, which is about $300 \mathrm{~km}$; for buses, the daily mileage is slightly less than that of taxis, which is about $200 \mathrm{~km}$.

\subsection{Parking Time of EVs}

The length of stay of EV users in a destination is very much related to the type of destination. When the user's travel destination is the work place, the general stay time may reach about 8 hours due to the work demand; when the user's travel destination is the leisure and entertainment place, the stay time may be only about 2 hours.

Fitting the data of the 2017 NHTS survey results, it is found that the residence time $t$ of private cars in the 
work area and other areas obey different generalized extreme value distributions respectively. Among them, the probability density of residence time in working area obeys the generalized extreme value distribution as follows:

$$
\left\{\begin{array}{l}
z=\frac{t-438.455}{164.506} \\
f(z)=\frac{1}{164.506} \exp \left[-(1-0.234 z)^{4.27}\right] \times(1-0.234 z)^{3.27}
\end{array}\right.
$$

The probability density of residence time in other regions obeys the generalized extreme value distribution as follows:

$$
\left\{\begin{array}{l}
z=\frac{t-68.520}{41.761} \\
f(z)=\frac{1}{41.761} \exp \left[-(1+0.657 z)^{-1.52}\right] \times(1+0.657 z)^{-2.52}
\end{array}\right.
$$

\subsection{Charging Methods of EVs}

According to the "Electric Vehicle Conductive Interface" adopted by my country in 2010 , the charging modes are divided into slow charging (charging mode L1), conventional charging (charging mode L2) and fast charging (charging mode L3). Among them, L1 and L2 are $\mathrm{AC}$ charging, $\mathrm{L} 2$ can be divided into three modes, and L3 is DC charging, as shown in the table below:

Table 1. Supply Mode of EVs.

\begin{tabular}{cccc}
\hline $\begin{array}{c}\text { Charging } \\
\text { Mode }\end{array}$ & $\begin{array}{c}\text { Rate } \\
\text { Voltage/V }\end{array}$ & $\begin{array}{c}\text { Rate } \\
\text { Current/A }\end{array}$ & $\begin{array}{c}\text { Applicable } \\
\text { Place }\end{array}$ \\
\hline L1 & 220 & 16 & Domestic \\
L2(2-1) & 220 & 32 & \\
L2(2-2) & 380 & 32 & Market \\
L2(2-3) & 380 & 63 & \\
L3 & 600 & 300 & $\begin{array}{c}\text { Charging } \\
\text { Station }\end{array}$ \\
\hline
\end{tabular}

\section{Travel Chain Model}

EVs are spatially mobile, and the uncertainty of the moving process will affect the spatial and temporal distribution prediction of electric vehicle charging power. Therefore, it is necessary to establish the spatial transfer model of EVs, and the travel chain can be used to describe the spatial transfer of electric vehicles in a day, including travel starting point and destination.

The travel locations of private cars and taxis have a typical purpose and can be described by a travel chain. Travel purposes can be divided into three types: home $(\mathrm{H})$, work $(\mathrm{W})$ and leisure $(\mathrm{O})$. These destinations are also charging places for EVs. There are three main types of travel chains involved in this paper, as shown in Figure 2.

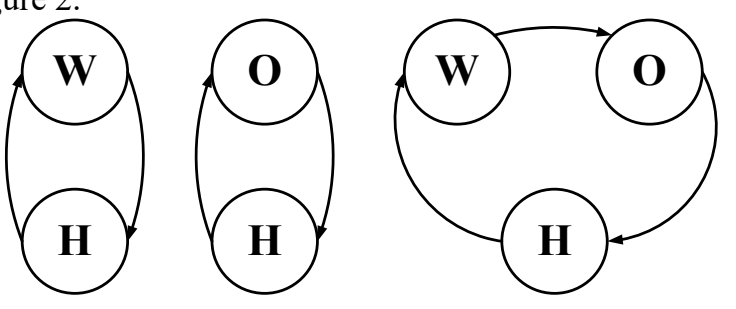

Figure.2. Typical travel chain structure.

According to the results of NHTS in 2017, the proportion of daily activity trips of private cars is as follows:

Table 2. Proportion of Different Travel Chains.

\begin{tabular}{cc}
\hline Travel Chains & Proportion\% \\
\hline H-W-H & 52.8 \\
H-O-H & 23.1 \\
H-W-O-H & 24.1 \\
\hline
\end{tabular}

\section{Calculation of EVs Charging Load}

Monte Carlo algorithm is a statistical simulation method that uses random numbers to solve practical problems[14]. In this paper, Monte Carlo algorithm is used to simulate and calculate the spatial and temporal distribution of EV charging load. According to the probability density distribution function, data such as the start time, mileage, travel chain and parking time are randomly extracted to obtain the charging demand of each EV in different communities, and the charging load variation curves of various functional areas are obtained by cumulative superposition.

The charging load of electric vehicle is calculated by Monte Carlo simulation method, and the simulation calculation process is shown in the following figure: 


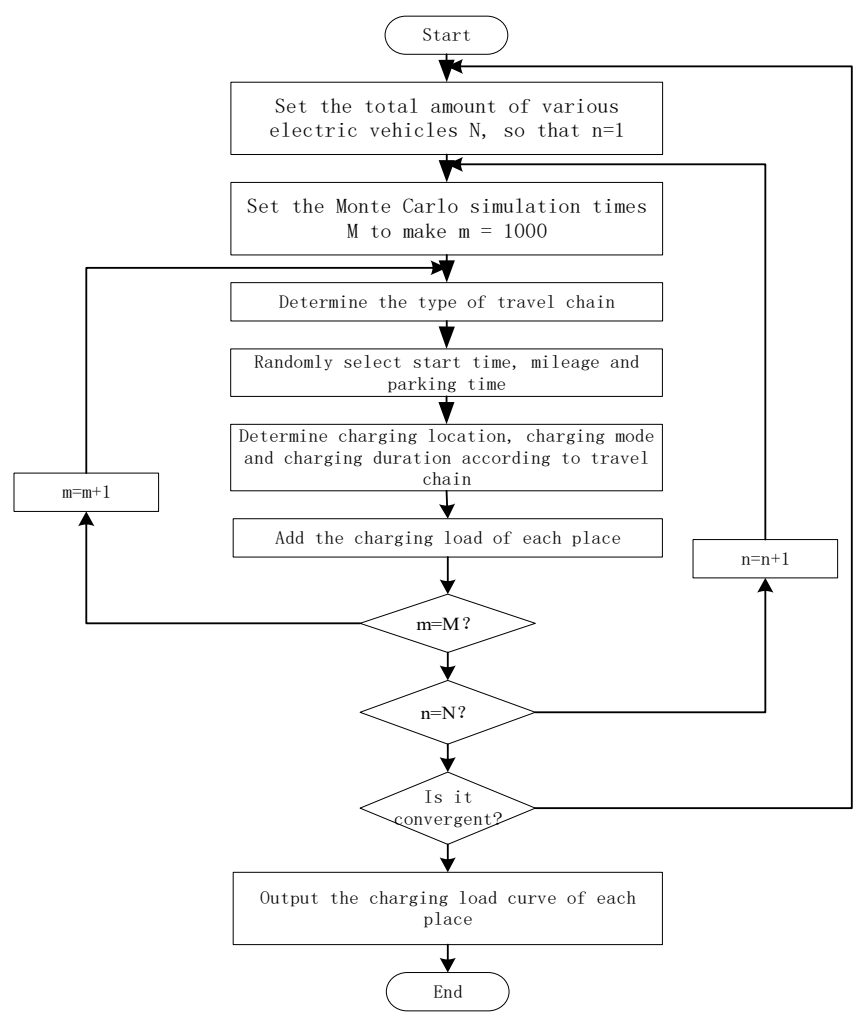

Figure.3. Flow chart of EV charging load calculation.

The specific calculation steps are as follows:

(1) Set the total number of various EVs, respectively set the number of private cars, taxis and buses in the area, and take $n=1$, to represent the simulation of the first $\mathrm{EV}$;

(2) Set the simulation times $M$ of Monte Carlo algorithm, and $M$ is taken as at least 1000 times in this paper.

(3) Based on the previous analysis, extract the travel chain type of the $n$-th EV;

(4) According to the probability density function obtained in the previous article, respectively extract the start time, the mileage and the length of parking;

(5) According to the type of travel chain, the charging place, charging mode and charging duration are determined, and the charging duration is not greater than the length of parking;

(6) Record the spatial and temporal distribution of the charging load of the $n$-th EV;

(7) Superimpose the charging load curve of each place, set $n=n+1$, if $n<N$, return to the third step to continue calculation;

(8) Define the variance coefficient of charging load $\beta_{i}$ :

$$
\beta_{i}=\frac{\theta_{i}(\bar{p})}{\sqrt{M} P_{i}}
$$

In the formula, $\theta_{i}(\bar{p})$ represents the standard deviation of the charging load at the $i$-th time, $P_{i}$ represents the expected value of the charging load at the $i$-th time, and $M$ represents the number of calculations. Require $\max \left(\beta_{i}\right) \leq 0.05 \%$.

\section{Example Analysis}

Assuming that the total number of electric private cars in a certain area is 20000 , the number of electric taxis is 10000 , and the electric buses is 12000 . According to the analysis above, the charging power of residential area is set at $3 \mathrm{~kW}$, the power of working area and commercial area is set as $14 \mathrm{~kW}$. It is assumed that the electric vehicles are fully charged at the beginning of the trip every day, that is, the initial SOC is 1 :

The EV charging load of residential area, working area and commercial area is obtained by Monte Carlo simulation, and superimposed with the traditional load of each functional area to obtain the prediction result of the space load, as shown in Figure 4-6. 


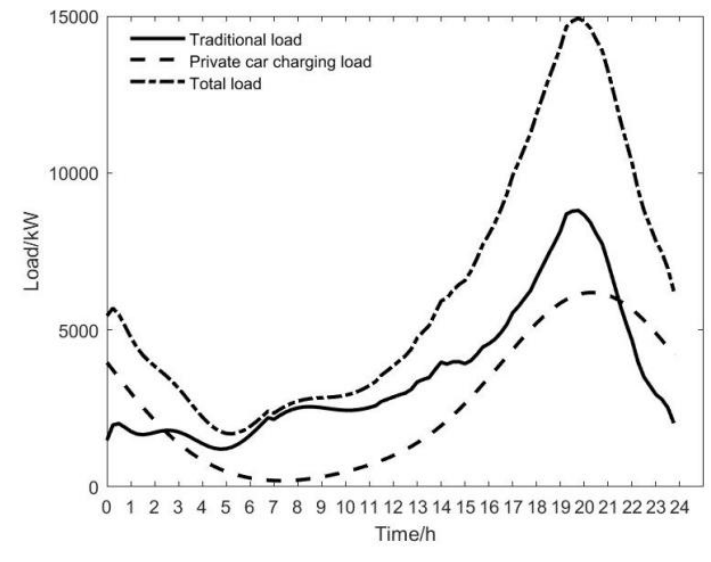

Figure.4. Daily Load of Residential Area.

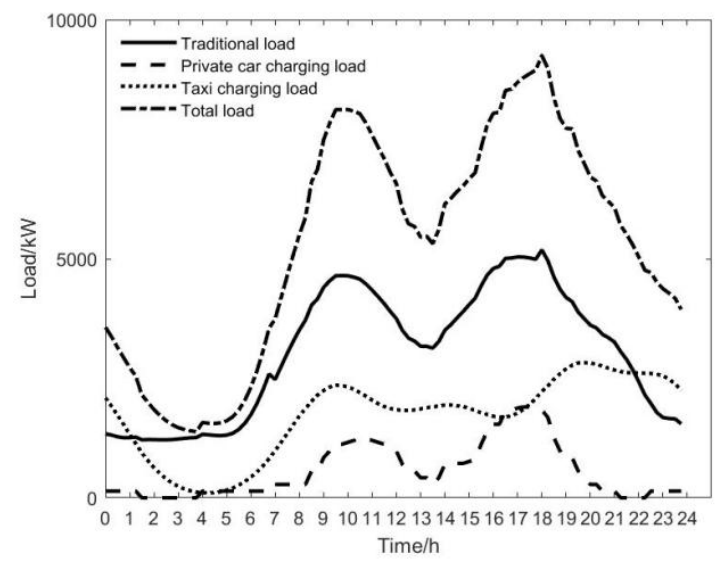

Figure.5. Daily Load of Working Area.

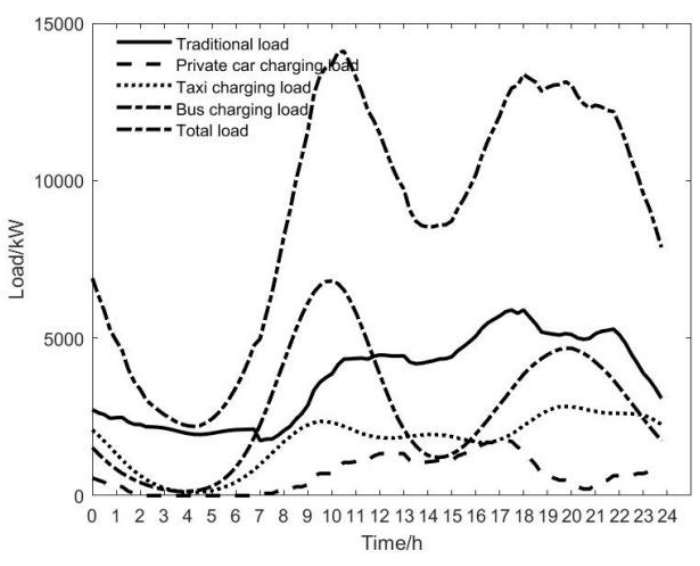

Figure.6. Daily Load of Commercial Area.

It can be seen from the figure that the charging behaviour of EVs in different functional areas are obviously different, and the charging behaviour of different types of EVs are also different. Due to the randomness of the driving path of private cars, it is necessary to supplement the power when arriving at the work area or commercial area during the day. At this time, fast charging is carried out. Therefore, the main charging load of private cars during the day is concentrated in the work area and the commercial area, while returning at night at home, in order to meet the travel needs of the next day, slow charging will be carried out, so the charging load of private cars at night is concentrated in residential areas; However, the daily driving mileage of taxis is long, and the battery capacity is not enough to meet the driving requirements of a day. Therefore, the taxis will be charged quickly during the operation, and will not be charged in residential areas. According to the choice of travel chain, the charging load is mainly concentrated in the working area and commercial area; Generally, the bus operation mode is double shifts. The charging of morning and afternoon buses will be managed and charged by the company. The charging places are commercial areas. Therefore, the charging load of buses has two peaks, which appear at 10:00 a.m. and 8:00 p.m. respectively.

\section{Conclusion}

Spatial load forecasting is extremely important to the planning and construction of distribution networks, and the large-scale access of EVs will affect the results of spatial load forecasting. In this paper, by studying he research on the spatial and temporal distribution of EV charging load, a spatial load forecasting method considering the spatial and temporal distribution of EV charging load is proposed. Aiming at different types of EVs, their respective occurrence rules are analyzed, and the travel chain model is established to simulate the spatial distribution of EVs, and the charging load of different functional areas is calculated by Monte Carlo method. The effectiveness of the method is verified by an example, and the necessity of considering the influence of EVs in urban distribution network spatial load forecasting is illustrated. The prediction results have a certain reference value for the future urban distribution network planning.

\section{References}

1. LEE WILLIS H, NORTHCOTE-GREEN J E D. (1983) Spatial electric load forecasting: a tutorial review. Proceedings of the IEEE. pp. 232-253. 
2. Liu, Z. F. Pang, C. C. Wang, Z. L. et al. (2013) Spatial load forecasting for distribution network based on cloud theory and cellular automata. Proceedings of the CSEE. pp. 98-105.

3. Zhou, Q. Deng, J. Y. Ren, H. J. et al. (2010) Research on spatial load forecast of distribution networks based on ant colony algorithm. Power System Protection and Control. pp. 99-104.

4. Li, J. Niu, C. D. X. Li, J. Y. et al. (2008) Spatial load forecasting based on load decomposition and support vector machine. Advanced Technology of Electrical Engineering and Energy. pp. 40-43.

5. Zhong, Q. Wu, J. Wu, L. et al. (2001) Subarea load forecasting based on system dynamics. Power System Technology. pp. 51-55.

6. Yu. R. (2001) Spatial load forecasting using nonuniform areas. International Electric Power for China. pp. 40-42.

7. Liu, Z. F. Pang, C. C. Wei, J. W. et al. (2012) Index calculation of load density based on IAHP and TOPSIS methods. Automation of Electric Power Systems. pp. 56-60.

8. Liu, Z. F. Chen, Y. X. Zhang, J. C. (2009) AHP and TOPSIS method based spatial electric load forecasting. Journal of Electric Power Science and Technology. pp. 44-48, 53.

9. Gao, C. Zhang, W. L. (2011) A survey of influence of electric vehicle charging on power grid. Power System Technology. pp. 127-131.

10. Wang, X. F. Shao, C. C. Wang, X. L. et al. (2013) Survey of electric vehicle charging load and dispatch control strategies. Proceeding of the CSEE. pp. 1-10.

11. WU Di, Aliprantis D C, Gkritza K. (2011) Electric energy and power consumption by lightduty plug-in electric vehicles. IEEE Transactions on Power Systems. pp.738-746.

12. Wu, Q. T. (2009) Progresses in "Ten Cities \& Thousand Units" plan. Automobile and Parts. pp.15-19.

13. Luo, Z. W. Hu, Z. C. Song, Y. H. et al. (2011) Study on plug-in electric vehicles charging load calculating. Automation of Electric Power Systems. pp. 36-42.

14. Tian, L. T. Shi, S. Jia, L. Z. (2010) A statistical model for charging power demand of electric vehicles. Power System Technology. pp. 126-130. 